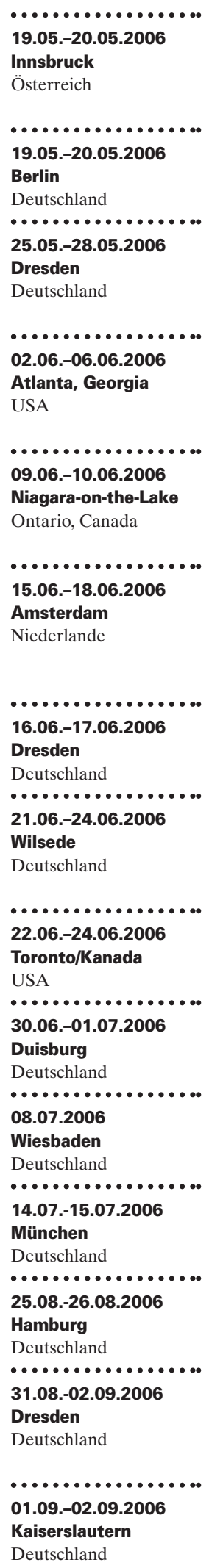

Konzepte und Therapieergebnisse Molekularbiologischer Tumortherapien

2. Interdisziplinärer Krebskongress

Das Mammakarzinom

12. Jahreskongress der Deutschen Gesellschaft für Radioonkologie (DEGRO)

\section{Asco Annual Meeting}

9. International Conference on Long-Term Complications of Treatment of Children \& Adolescents for Cancer

11. Congress of the European Hematology Association (EHA)

UPDATE Hämatologie / Onkologie 2006

Wilsede-Schule für Onkologie und Hämatologie:

Myelom - CUP - Pharmakogenetik/-genomie

18. International Symposium on Supportive Care in Cancer

UPDATE Hämatologie / Onkologie 2006

GI-Oncology 2006 - 2. Interdisziplinäres Update

UPDATE Hämatologie / Onkologie 2006

UPDATE Hämatologie / Onkologie 2006

26. Jahrestagung der Deutschen Gesellschaft für Senologie

178. Tagung der Mittelrheinischen Gesellschaft für Gynäkologie und Geburtshilfe
Auskunft: European School of Oncology - deutschsprachiges Programm (ESO-d), CH-St. Gallen E-mail ESO-d@sg.zetup.ch

www.zetup.ch

Auskunft: RRC-Congress GmbH, Berlin

E-mail info@rrc-congress.de

www.rrc-congress.de

Auskunft: Deutsche Gesellschaft für Radioonkologie e.V., Berlin

Tel. +49 3084419188

E-mail office@degro.org

Auskunft: American Society of Clinical Oncology,

Auskunft: American

E-mail asco@asco.org

www.asco.org

Auskunft: Daniel Green, MD, Pediatrics, Roswell

Park Cancer Institute, Buffalo, NY

Tel. (716) 845-2334, Fax (716) 845-8003

E-mail daniel.green@roswellpark.org

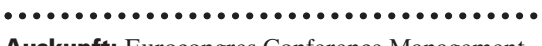

Auskunft: Eurocongres Conference Management,

NL-Amsterdam

Tel. +31 206793411, Fax +31206737306

E-mail: eha@eurocongres.com

www.eurocongres.com/eha2006/

Auskunft: Interplan AG, München

E-mailinfo@interplan.de

www.interplan.de

Auskunft: European School of Oncology - deutschsprachiges Programm (ESO-d), CH-St. Gallen

E-mail ESO-d@sg.zetup.ch

www.zetup.ch oder www.wilsede-schule.de

•.........................

Auskunft: www.mascc.org

Auskunft: Interplan AG, München

E-mail info@interplan.de

www.interplan.de

Auskunft: EMC Event \& Meeting Company GmbH

E-mail fronmueller@emc-event.com

www.kongresseonline.de/gi-oncology

Auskunft: Interplan AG, München

E-mail info@interplan.de

www.interplan.de

Auskunft: Interplan AG, München

E-mailinfo@interplan.de

www.interplan.de

-..................

Auskunft: CTW - Congress Organisation Thomas

Wiese GmbH, 14199 Berlin

E-mail senologie@ctw-congress.de

www.senologiekongress.de

Auskunft: COCS - Congress Organisation

C. Schäfer, München

E-mail katrin.lehmann@cocs.de

www.cocs.de 
06.09.-09.09.2006

Wilsede

Deutschland

\subsection{9.-09.09.2006}

Salzburg

Österreich

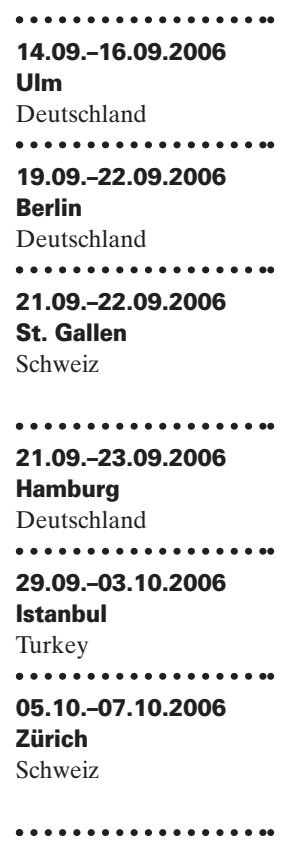

05.10.-07.10.2006

St. Wolfgang

Österreich

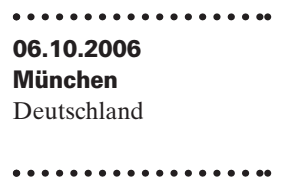

04.11.-08.11.2006
Leipzig
Deutschland

\section{$06.11 .-08.11 .2006$ \\ Wien}

Österreich

15.11.-16.11.2006
Klagenfurt
Österreich
$\begin{aligned} & \text { 23.11.-25.11.2006 } \\ & \text { Wien }\end{aligned}$

Österreich

\subsection{2.-12.12.2006}

Orlando, Florida

USA

21.0...............

Dresden

Deutschland
Wilsede-Schule für Onkologie und Hämatologie:

Mikroskopierkurs für Anfänger - Thrombophilie bei Tumorpatienten

23. Jahrestagung der Österreichischen Gesellschaft für Senologie

12. Congress of the European Shock Society (ESS)

56. Kongress der Deutschen Gesellschaft für Gynäkologie und Geburtshilfe

10. Internationales ESO-D Jubiläums-Seminar:

"Onkologische Krankenpflege - Fortgeschrittene Praxis"

6. Kongress der Deutschen Gesellschaft für Palliativmedizin

"Teamarbeit und Kommunikation"

\section{ESMO 2006}

31. ESMO Congress

Multidisziplinäre Behandlung Thorakaler Tumoren

23. Jahrestagung der Österreichischen Gesellschaft für Chirurgische Onkologie (ACO-ASSO)

11. International Thoracic Surgery Congress

Geriatrische Probleme der Hämatologie und Onkologie

Gemeinsame Jahrestagung der Deutschen, Österreichischen und Schweizerischen Gesellschaften für Hämatologie und Onkologie

Onkologie in Klinik und Praxis

Interdisziplinäre Behandlungsstrategien von HNO-Tumoren (im Rahmen der ÖGRO-Jahrestagung)

Methodik Klinischer Prüfung in der Onkologie

American Society for Hematology (ASH):

48. Annual Meeting

51. Jahrestagung der Gesellschaft für Thrombose- und

Hämostaseforschung e.V.

51. Annual Meeting Society of Thrombosis and Haemostasis Research
Auskunft: European School of Oncology - deutschsprachiges Programm (ESO-d), CH-St. Gallen E-mail ESO-d@sg.zetup.ch

www.zetup.ch oder www.wilsede-schule.de

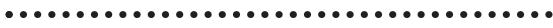

Auskunft: CTW - Congress Organisation Thomas

Wiese GmbH, Berlin

E-mail info@ctw-congress.de

www.ctw-congress.de/oegs

Auskunft: comed GmbH, Köln

E-mailinfo@comed-kongresse.de

www.comed-kongresse.de

Auskunft: AAK GmbH, Düsseldor

E-mail info@aakongress.de

www.aakongress.de

•............................

Auskunft: European School of Oncology - deutschsprachiges Programm ESO-d, CH-St. Gallen

Tel. +41 71-243 0032, Fax -245 6805

E-mail eso-d@sg.zetup.ch

Auskunft: Interplan AG, München

E-mailinfo@interplan.de

www.interplan.de

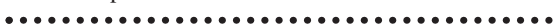

Auskunft: ESMO Head Office, $\mathrm{CH}$-Viganello-Lugano

E-mail congress@esmo.org

www.esmo.org

Auskunft: European School of Oncology - deutschsprachiges Programm (ESO-d), CH-St. Gallen

E-mail ESO-d@sg.zetup.ch

www.zetup.ch

Auskunft: Sylvia Tittinger, Wiener Medizisische

Auskunft: Sylvia Trittinger, Wiener Medizinische
Akademie, $\mathrm{CH}-$ Wien

E-mail st@medacad.org

www.medacad.org

................................

Auskunft: European School of Oncology - deutsch

sprachiges Programm (ESO-d), CH-St. Gallen

E-mail ESO-d@sg.zetup.ch

www.zetup.ch oder www.klinik-wartenberg.de

Auskunft: Hämatologie-Onkologie 2006

c/o AKM Congress Service, CH-Basel

E-mail info@akm.ch

www.akm.ch/haematologie-onkologie06

..................................

Auskunft: European School of Oncology - deutsch-

sprachiges Programm (ESO-d), CH-St. Gallen

E-mail ESO-d@sg.zetup.ch

www.zetup.ch oder www.onkologie.at

•.............................

Auskunft: European School of Oncology - deutsch-

sprachiges Programm (ESO-d), CH-St. Gallen

E-mail ESO-d@sg.zetup.ch

www.zetup.ch oder www.oegroklagenfurt2006.at

Auskunft: European School of Oncology - deutsch-

sprachiges Programm (ESO-d), CH-St. Gallen

E-mail ESO-d@sg.zetup.ch

www.zetup.ch oder www.cesar.or.at

Auskunft: ASH Headquarters, Washington D.C., USA

E-mail ash@hematology.org

www.hematology.org

Auskunft: Congress Partner $\mathrm{GmbH}$, Berlin

Tel. +49 30 20459-0, Fax -50

E-mail berlin@cpb.de 